\title{
Loop Transfer Recovery Techniques for Discrete-Time Non-Minimum Phase Plants with Feedback Delays
}

\author{
Tadashi ISHIHARA \\ Graduate School of Information Sciences, Tohoku University, \\ Aramaki aza Aoba, Aoba-ku, Sendai, 980-77, Japan
}

Received July 17, 1995; final version accepted November 1, 1995

\begin{abstract}
For discrete-time non-minimum phase plants with feedback delays, we discuss two Loop Transfer Recovery (LTR) techniques applied at the plant output side. We show that the feedback property achieved by the formal application of the conventional LTR procedure can be achieved by the partial LTR technique which has a clear system-theoretic significance. By this fact, we can justify the use of the conventional LTR technique to a class of non-minimum phase plants. In addition, we point out that the partial LTR technique provides more freedom in shaping a target feedback property via the selection of disturbance model. We propose a simple technique exploiting this freedom. By a numerical example, we show that the proposed technique can be used to enhance robustness against uncertainties near a Nyquist frequency without significant change in low frequency characteristics.
\end{abstract}

KEYWORDS: Discrete-time systems, Non-minimum phase systems, Feedback delays, LQG controller, Loop transfer recovery

\section{INTRODUCTION}

Digital control systems contain feedback delays such as computation delays in digital processors, and sensor and actuator signal delays (e.g., Halevi and Ray, 1988). Ishihara (1988) has discussed the application of loop transfer recovery (LTR) techniques (Kwakernaak, 1969; Doyle and Stein, 1979) to minimum phase discrete-time systems with feedback delays. However, the results of his study were somewhat inadequate since a discrete-time model obtained by discretization with a zeroth order holder is frequently non-minimum phase even if a continuous-time plant model is minimum-phase (Astrom et al., 1984).

Several researchers have already discussed the formal application of the conventional LTR technique to nonminimum phase plants. For a discrete-time LQG control system with a filtering type or a prediction type Kalman filter, Maciejowski (1985) has suggested that the LTR technique is still useful for non-minimum phase plants to recover a target feedback property in the frequency region where the effect of unstable zeros is negligible. For the continuous-time case, Zhang and Freudenberg (1990) have justified this observation in a more rigorous setting. Furthermore, they have considered a discrete-time version for LQG controllers using either type of Kalman filter (Zhang and Freudenberg, 1991). Recently, Saberi et al. (1991) have characterized recoverable targets by using a geometric approach. Although these results are worthwhile, no simple system-theoretic interpretation has been given to the feedback property achieved by formal application of the LTR procedure.

For the continuous-time case, Moore and Xia (1987) have proposed a partial LTR technique which has the clear system-theoretic intention to recover only a part of the feedback property related to the minimum phase part of a plant. Note that this technique can be regarded as a Riccati equation formalism to accomplish the subperfect regnlation (Kimura, 1982) which is a plausible design procedure for non-minimum phase plants. However, the relation to formal application of the conventional LTR procedure has not been discussed in the iramework of Riccati equation formalism.

In this paper, we clarify the relation between the two LTR techniques for discrete-time non-minimum phase plants with feedback delays. We show that the feedback property achieved by enforcing the conventional LTR procedure can be achieved by the partial LTR technique. In addition, we point out that the partial LTR technique provides more design freedom in shaping a target feedback property. We propose a simple technique for exploiting this design freedom.

Although LTR procedures are dual for a plant output and a plant input, except for the delayless case, we discuss the plant output case for which more intricate discussion is required to give a clear system-theoretic interpretation for the achievable feedback property. Since loop transfer function matrices for our case are rather complicated, we use decompositions of sensitivity matrices to simplify discussion. 


\section{PREDICTOR-BASED LQG CONTROLLER}

Consider a discrete-time plant described by

$$
x(t+1)=A x(t)+B u(t)+w(t), \quad y(t)=C x(t)+v(t),
$$

where $x(t) \in R^{n}$ is a state vector, $u(t) \in R^{p}$ is a control input vector, $y(t) \in R^{p}$ is an output vector, $w(k)$ and $v(k)$ are mutually independent zero-mean white noise processes with covariance matrices $Q$ and $R$, respectively. We assume that the pairs $(A, B)$ and $(C, A)$ are controllable and observable, respectively, and that the matrix $C B$ is non-singnlar. In addition, we assume that feedback path for the plant (2.1) contains $L$-step delays.

Introduce a quadratic performance index defined as

$$
V^{\infty}=\lim _{T \rightarrow \infty} E\left\{\frac{1}{T} \sum_{t=0}^{T-1}\left[y^{\prime}(t) Q_{c} y(t)+u^{\prime}(t+L) R_{c} u(t+L)\right]\right\},
$$

where $Q_{c}>0$ and $R_{c} \geq 0$. The optimal control input for (2.1) under (2.2) is given by

$$
u(t+L)=-F \hat{x}(t+L \mid t),
$$

where $F$ is the optimal feedback gain matrix for the delay free case and $\hat{x}(t+L \mid t)$ is the optimal $L$-step ahead prediction of the state based on the data available up to time $t$.

The optimal feedback gain matrix is given by

$$
F \triangleq\left(B^{\prime} P_{c} B+R_{c}\right)^{-1} B^{\prime} P_{c} A,
$$

where $P_{c}$ is a non-negative definite solution of the matrix Riccati equation

$$
P_{c}=A^{\prime} P_{c} A-A^{\prime} P_{c} B\left(B^{\prime} P_{c} B+R_{c}\right)^{-1} B^{\prime} P_{c} A+C^{\prime} Q_{c} C .
$$

The prediction for $L=1$ can be directly obtained by a prediction type Kalman filter

$$
\hat{x}(t+1 \mid t)=A \hat{x}(t \mid t-1)+B u(k)+K[y(t)-C \hat{x}(t \mid t-1)],
$$

where $K$ is the optimal filter gain matrix

$$
K \triangleq A P C^{\prime}\left(C P C^{\prime}+R\right)^{-1}
$$

with $P$ being a non-negative definite solution of the matrix Riccati equation

$$
P=A P A^{\prime}-A P C^{\prime}\left(C P C^{\prime}+R\right)^{-1} C P A^{\prime}+Q .
$$

For $L>1$, we can obtain the prediction by connecting the $(L-1)$-step state predictor

$$
\hat{x}(t+L \mid t)=A^{L-1} \hat{x}(t+1 \mid t)+A^{L-2} B u(t+1)+\cdots+B u(t+L-1)
$$

to the Kalman filter (2.6).

For the sensitivity matrix at the plant output, we have the following decomposition, which can easily be obtained by the technique used in Ishihara et al. (1992).

Lemma 2.1: Consider the control system consisting of the plant (2.1) and the predictor-based LQG controller (2.3). Let $S_{L}(z)$ denote the sensitivity matrix at the plant output. Then the sensitivity matrix can be expressed as

$$
S_{L}(z)=\left[W_{L-1}(z)+z^{-(L-1)} C(z I-A+B F)^{-1} A^{L-1} K\right] S(z),
$$

where

$$
S(z)=\left[I+C(z I-A)^{-1} K\right]^{-1}
$$

is the sensitivity matrix of the Kalman filter and

$$
W_{L-1}(z)=\left\{\begin{array}{l}
I \quad(L=1) \\
I+z^{-1} C K+z^{-2} C A K+\cdots+z^{-(L-1)} C A^{L-2} K \quad(L \geq 2) .
\end{array}\right.
$$

\section{FORMAL APPLICATION OF LTR TECHNIQUE}

Since we adopt Riccati equation formalism as a recovery procedure, we choose the weighting matrices in the quadratic performance index (2.2) as

$$
Q_{c}=\rho I, \quad R_{c}=I,
$$

where $\rho$ is a positive scalar and $I$ is an identity matrix. We are interested in the feedback property at the plant out- 
put achieved by letting $\rho$ tend to infinity. To obtain an expression for the achievable sensitivity matrix, we assume that the plant transfer function matrix $G(z)=C(z I-A)^{-1} B$ can be decomposed as

$$
G(z)=G_{a}(z) G_{m}(z)
$$

where $G_{a}(z)$ is all-pass part satisfying

$$
G_{a}^{\prime}\left(z^{-1}\right) G_{a}(z)=I .
$$

and $G_{m}(z)$ is minimum phase part which can be taken as

$$
G_{m}(z)=C_{m}(z I-A)^{-1} B,
$$

where the matrix $C_{m}$ is an appropriate matrix. The above decomposition can be obtained in various ways. A method based on the discrete-time version of the continuous-time result obtained by Enns (1988) will be discussed in Section 5 .

From the above decomposition, the following result for the optimal feedback gain matrix can easily be obtained (e.g., Shaked, 1985).

Lemma 3.1: Gonsider the optimal control problem for $(A, B, C)$ under the quadratic performance index (2.2) with the weighting matrices (3.1). Let $\rho$ tend to infinity. Then the optimal feedback gain approaches

$$
F^{*}=\left(C_{m} B\right)^{-1} C_{m} A \text {. }
$$

Substituting (3.5) into the expression (2.10), we have the following result for the sensitivity achieved by enforcing the LTR procedure.

Proposition 3.1: Gonsider the predictor-based LQG controller (2.3) for the plant (2.1) and the quadratic performance index (2.2) with the weighting matrices (3.1). Let $q$ tend to infinity. Then the sensitivity matrix at the plant output approaches

$$
S_{L}^{*}(z)=\left\{\tilde{W}_{L}(z)+\left[C-G_{a}(z) C_{m}\right](z I-A)^{-1} K\right\} S(z),
$$

where

$$
\tilde{W}_{L}(z)=I+z^{-1} G_{a}(z) C_{m} K+z^{-2} G_{a}(z) C_{m} A K+\cdots+z^{-L} G_{a}(z) C_{m} A^{L-1} K \quad(L \geq 1) .
$$

\section{PARTIAL LTR TECHNIQUE}

In this section, we discuss an application of the partial LTR technique at the plant output. First, we construct an LQG controller that depends only on the estimate of the minimum phase state. i.e.,

Let $\left\{A_{a}, B_{a}, C_{a}, D_{a}\right\}$ denote the state space representations of the all pass part $G_{a}(z)$ defined in Lemma 3.1,

$$
G_{a}(z)=C_{a}\left(z I-A_{a}\right)^{-1} B_{a}+D_{a} .
$$

We also define the state vectors of the all-pass part and the minimum-phase part as $x_{a}(t)$ and $x_{m}(t)$, respectively. Noting that the minimum phase part is described by $\left(A, B, C_{m}\right)$, we can construct a state space stochastic model of the plant as

$$
\chi(t+1)=\Phi \chi(t)+\Gamma u(t)+\Omega w(t), \quad y(t)=H \chi(t)+v(t),
$$

where $x(t)$ is a state vector defined as

$$
\chi(t)=\left[x_{m}^{\prime}(t) x_{a}^{\prime}(t)\right]^{\prime},
$$

and

$$
\Phi=\left[\begin{array}{cc}
A & 0 \\
B_{a} C_{m} & A_{a}
\end{array}\right], \quad \Gamma=\left[\begin{array}{c}
B \\
0
\end{array}\right], \quad \Omega=\left[\begin{array}{l}
I \\
0
\end{array}\right], \quad H=\left[\begin{array}{ll}
D_{a} C_{m} & C_{a}
\end{array}\right]
$$

\section{MINIMUM PHASE ESTIMATOR FEEDBACK CONTROLLER}

For the model described by (4.2), we can design an LQG controller taking account of $L$-step feedback delays. As discussed by Moore and Xia (1987) for the continuous-time case, the partial LTR at the plant input is achieved by increasing the covariance matrix of the fictitious disturbances inserted between the all-pass part and the minimum phase part. To keep the discussion dual to the recovery at the plant input, we introduce the fictitious output vector 


$$
y_{f}(t)=C_{f} \chi(t)=\left[\begin{array}{cc}
C_{m} & 0 \\
D_{a} C_{m} & C_{a}
\end{array}\right]\left[\begin{array}{l}
x_{m}(t) \\
x_{a}(t)
\end{array}\right]
$$

for the partial LTR at the plant output. Note that the fictitious output vector consists of the actual output of the plant and the output of the minimum phase part. We define a quadratic performance index consisting of the fictitious output vector and the control vector as

$$
V_{f}^{\infty}=\lim _{T \rightarrow \infty} E\left\{\frac{1}{T} \sum_{t=0}^{T-1}\left[y_{f}^{\prime}(t) Q_{f} y_{f}(t)+u^{\prime}(t+L) R_{c} u(t+L)\right]\right\},
$$

where

$$
Q_{f}=\operatorname{diag}\left[\begin{array}{ll}
Q_{m} & Q_{a}
\end{array}\right]
$$

and $Q_{f} \geq 0$ and $R_{c}>0$.

The optimal control law minimizing the performance index (4.6) is given by

$$
u(t+L)=-F_{f} \hat{x}(t+L \mid t),
$$

where $F_{f}$ is the optimal feedback gain matrix for the delay-free case, i.e., $L=0$ and $\hat{x}(t+L \mid t)$ is the optimal $L$ step projected prediction of the state based on the data available up to time $t$. As discussed in Section 2, the LQG controller consists of the optimal feedback gain matrix, the state predictor and the Kalman filter.

\section{A. Feedback Gain Matrix}

The optimal feedback gain matrix $F_{f}$ for $L=0$ is given by

$$
F_{f}=\left(R_{c}+\Gamma^{\prime} \Pi_{c} \Gamma\right)^{-1} \Gamma^{\prime} \Pi_{c} \Phi,
$$

where $\Pi_{c}$ is a positive semi-definite solution of the algebraic Riccati equation

$$
\Pi_{c}=\Phi^{\prime} \Pi_{c} \Phi-\Phi^{\prime} \Pi_{c} \Gamma\left(R_{c}+\Gamma^{\prime} \Pi_{c} \Gamma\right)^{-1} \Gamma^{\prime} \Pi_{c} \Phi+C_{f}^{\prime} Q_{f} C_{f}
$$

For the feedback gain matrix given by (4.9), we can obtain the following result, which corresponds to Lemma 1 in Xia and Moore (1987).

Lemma 4.1: Assume that $Q_{m}=\rho_{m} I$ and $Q_{a}=\rho_{a} I$ where $\rho_{m}$ and $\rho_{a}$ are non-negative scalars. Then the submatrices of the obvious partition of $\Pi_{c}$ satisfy the relations

$$
\begin{aligned}
\Pi_{m m}= & A^{\prime} \Pi_{m m} A-A^{\prime} \Pi_{m m} B\left(R_{c}+B^{\prime} \Pi_{m m} B\right)^{-1} B^{\prime} \Pi_{m m} A \\
& +C_{m}^{\prime}\left(q_{m} I+q_{a} D_{a}^{\prime} D_{a}+B_{a}^{\prime} \Pi_{a a} B_{a}\right) C_{m}, \\
\Pi_{a a}= & A_{a}^{\prime} \Pi_{a a} A_{a}+q_{a} C_{a}^{\prime} C_{a}, \quad \Pi_{m a}=\Pi_{a m}^{\prime}=0 .
\end{aligned}
$$

In addition, the feedback gain matrix (4.9) can be expressed as

$$
F_{f}=\left[\begin{array}{ll}
F_{m} & F_{a}
\end{array}\right] \text {, }
$$

with $F_{a}=0$ and $F_{m}$ given by

$$
F_{m}=\left(R_{c}+B^{\prime} \Pi_{m m} B\right)^{-1} B^{\prime} \Pi_{m m} A
$$

\section{B. Kalman Filter}

The prediction type Kalman filter for the plant described by (4.2)-(4.4) is given by

$$
\hat{\chi}(t+1 \mid t)=\Phi \hat{\chi}(t \mid t-1)+\Gamma u(t)+\Lambda[y(t)-H \hat{\chi}(t \mid t-1)],
$$

where $\Lambda$ is a Kalman filter gain matrix defined by

$$
\Lambda \triangleq \Phi \Pi H^{\prime}\left(H \Pi H^{\prime}+R\right)^{-1},
$$

with $\Pi$ being a non-negative definite solution of the matrix Riccati equation

$$
\Pi=\Phi \Pi \Phi^{\prime}-\Phi \Pi H^{\prime}\left(H \Pi H^{\prime}+R\right)^{-1} H \Pi \Phi^{\prime}+\Omega Q \Omega^{\prime}
$$

Using the partitions

$$
\hat{\chi}(t \mid t-1)=\left[\begin{array}{c}
\hat{x}_{m}(t \mid t-1) \\
\hat{x}_{a}(t \mid t-1)
\end{array}\right], \quad \Lambda=\left[\begin{array}{c}
K_{m} \\
K_{a}
\end{array}\right],
$$

we can decompose the algorithm (4.15) into 


$$
\begin{aligned}
& \hat{x}_{a}(t+1 \mid t)=A_{a} \hat{x}_{a}(t \mid t-1)+B_{a} C_{m} \hat{x}_{m}(t \mid t-1)+K_{a}\left[y(t)-D_{a} C_{m} \hat{x}_{m}(t \mid t-1)-C_{a} \hat{x}_{a}(t \mid t-1)\right], \\
& \hat{x}_{m}(t+1 \mid t)=A \hat{x}_{m}(t \mid t-1)+B u(t)+K_{m}\left[y(t)-D_{a} C_{m} \hat{x}_{m}(t \mid t-1)-C_{a} \hat{x}_{a}(t \mid t-1)\right] .
\end{aligned}
$$

Taking z-transforms of both sides of (4.19) and (4.20), we can write the z-transform of the estimate of the minimum-phase state vector as

$$
\hat{x}_{m}(z)=\left[z I-A+K(z) G_{z}(z) C_{m}\right]^{-1}[B u(z)+K(z) y(z)],
$$

where

$$
K(z)=K_{m}\left[I+C_{a}\left(z I-A_{a}\right)^{-1} K_{a}\right]^{-1} .
$$

Using (4.21) and (4.22), we can obtain the estimate of the minimum-phase state without computing that of the all-pass state vector. Note that the filter gain matrix given by (4.22) is frequency-shaped.

\section{State Predictor}

The optimal $L$-step ahead predictor of $x(t)$ based on the data available up to time $t$ is given by

$$
\hat{\chi}(t+L \mid t)=\Phi^{L-1} \hat{\chi}(t+1 \mid t)+\Phi^{L-2} \Gamma u(t+1)+\cdots+\Gamma u(t+L-1) .
$$

Using (4.4) in (4.23), we can write the predictor for the minimum-phase state vector as

$$
\hat{x}_{m}(t+L \mid t)=A^{L-1} \hat{x}_{m}(t+1 \mid t)+A^{L-2} B u(t+1)+\cdots+B u(t+L-1) .
$$

From the above results, we can simplify the optimal control law (4.8) as

$$
u(t+L)=-F_{m} \hat{x}_{m}(t+L \mid t)
$$

which only depends on the estimate of the minimum-phase state vector. We call this controller the minimumphase estimate feedback (MEF) controller. The structure of the MEF controller is shown in Fig. 1.

\section{PARTIAL RECOVERY}

To discuss partial LTR, we use the following result.

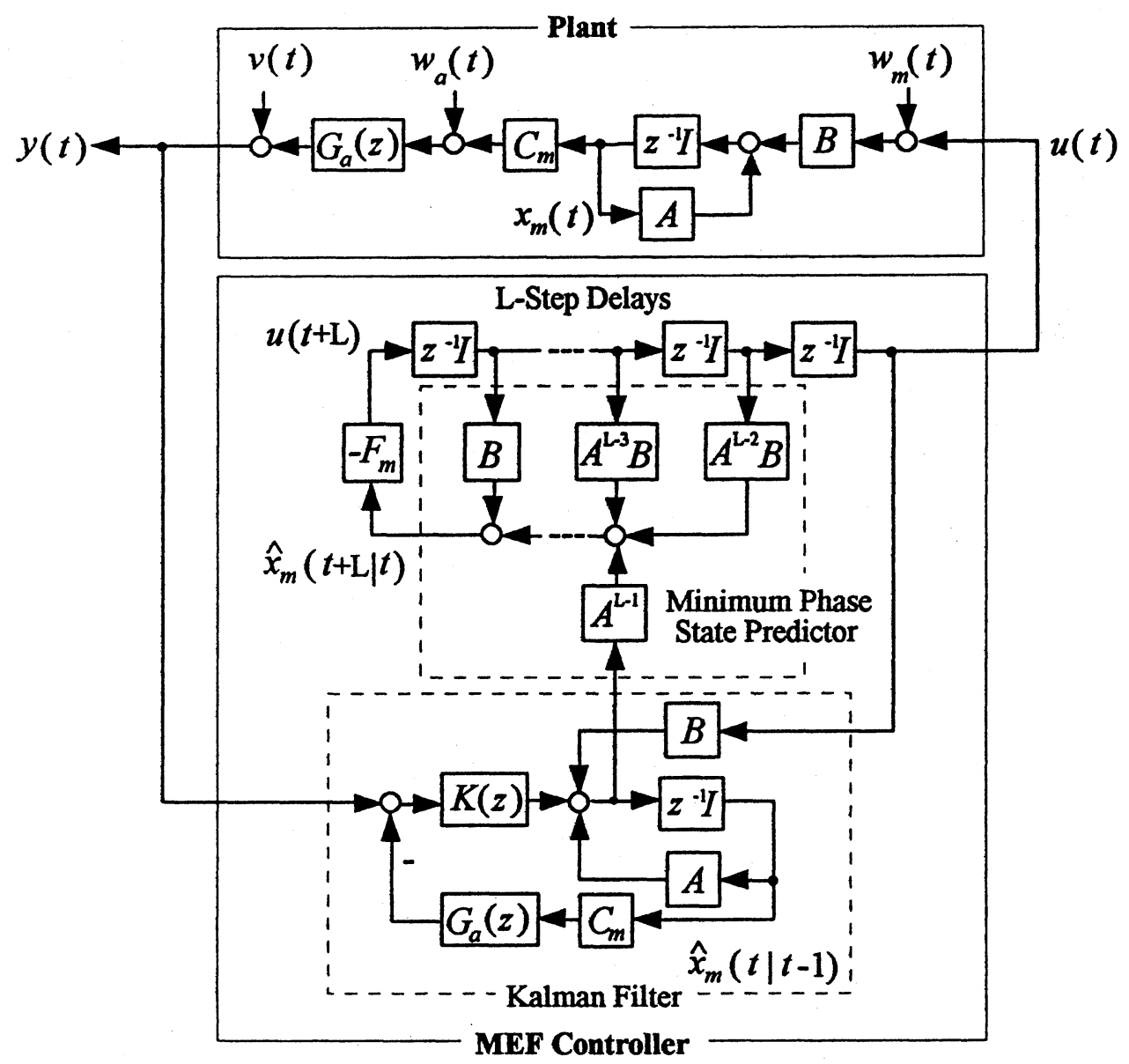

Fig. 1 Structure of the MEF controller. 
Lemma 4.2: Consider the control system shown in Fig. 1. The sensitivity matrix at the output of the plant can be expressed as

$$
\begin{aligned}
\Sigma_{L}(z)= & {\left[\bar{W}_{L-1}(z)+z^{-(L-1)} G_{a}(z) C_{m}\left(z I-A+B F_{m}\right)^{-1} A^{L-1} K(z)\right] } \\
& \times\left[I+G_{a}(z) C_{m}(z I-A)^{-1} K(z)\right]^{-1}
\end{aligned}
$$

where

$$
\bar{W}_{L-1}(z)=\left\{\begin{array}{l}
I \quad(L=1) \\
I+G_{a}(z) C_{m}\left(z^{-1} I+\cdots+z^{-(L-1)} A^{L-2}\right) K(z) \quad(L \geq 2) .
\end{array}\right.
$$

Proof: The decomposition of the sensitivity matrix given in (4.26) can be obtained by direct but tedious matrix calculations. An easy way is to use the fact that the derivation of Lemma 2.1 still valid for frequency-shaped versions of the matrices. By replacing the output matrix $C$ and filter gain matrix $K$ in Lemma 2.1 by $C_{m} G_{a}(z)$ and $K(z)$, respectively, we have the decomposition (4.26).

Using the above result, we can identify the sensitivity matrices achieved by the partial loop recovery technique.

Proposition 4.1: Consider the MEF controller minimizing the performance index (4.6) with the weighting matrix

$$
Q_{f}=\operatorname{diag}\left[\rho_{m} I \rho_{a} I\right]
$$

where $\rho_{m}$ and $\rho_{a}$ are non-negative scalars. Then, as $\rho_{m}$ tends to infinity, the sensitivity matrix at the plant output approaches

$$
\Sigma_{L}^{*}(z)=\lim _{\rho \mathrm{m} \rightarrow \infty} \Sigma_{L}(z)=\bar{W}_{L}(z)\left[I+G_{a}(z) C_{m}(z I-A)^{-1} K(z)\right]^{-1}
$$

Proof: Using the matrix defined in (4.29), we can rewrite (4.26) as

$$
\begin{aligned}
\Sigma_{L}(z)= & \Sigma_{L}^{*}(z)+z^{-L} G_{a}(z) C_{m}\left(A-B F_{m}\right)\left(z I-A+B F_{m}\right)^{-1} \\
& \times A^{L-1} K(z)\left[I+G_{a}(z) C_{m}(z I-A)^{-1} K(z)\right]^{-1},
\end{aligned}
$$

Employing the result of Shaked (1985) in (4.11), (4.12) and (4.14), we can check that, as the weight $\rho_{m}$ tends to infinity, the feedback gain matrix $F_{m}$ for the minimum-phase part approaches

$$
F_{m}^{*}=\left(C_{m} B\right)^{-1} C_{m} A \text {. }
$$

Substituting (4.31) into (4.30), we can easily obtain (4.29).

Although the above result gives an explicit representation for the sensitivity matrix achieved by the partial loop recovery technique, it does not provide clear system-theoretic meaning for the recovered sensitivity matrix.

\section{RELATED ESTIMATION PROBLEM}

To give a clear system-theoretic interpretation for the sensitivity matrix (4.5), we consider an estimation problem with $L$-step delays in the observation process. Assume that a process to be estimated and the observation process are described by

$$
\chi(t+1)=\Phi \chi(t)+\Omega w(t), \quad y_{L}(t)=H \chi(t-L)+v(t)
$$

where the matrices $\Phi, \Omega$ and $H$ are defined in (4.4). To construct an estimator, we define an extended system as

$$
\chi_{L}(t+1)=\Phi_{L} \chi_{L}(t)+\Omega_{L} w(t), \quad y_{L}(t)=H_{L} \chi_{L}(t)+v(t),
$$

where

$$
\begin{aligned}
& \chi_{L}(t)=\left[\chi^{\prime}(t) \chi^{\prime}(t-1) H^{\prime} \chi^{\prime}(t-2) H^{\prime} \cdots \chi^{\prime}(t-L) H^{\prime}\right]^{\prime} \\
& \Phi_{L}=\left[\begin{array}{cccccc}
\Phi & 0 & \cdot & \cdot & \cdot & 0 \\
H & 0 & \cdot & . & \cdot & 0 \\
0 & I & 0 & \cdot & \cdot & 0 \\
& \cdot & \cdot & . & . & \\
0 & . & . & . & I & 0
\end{array}\right], \quad \Omega_{L}=\left[\begin{array}{c}
\Omega \\
0 \\
0 \\
\vdots \\
0
\end{array}\right], \quad H_{L}=\left[\begin{array}{lll}
0 & \cdots & I
\end{array}\right]
\end{aligned}
$$

For the extended system (4.33), we can construct the prediction type Kalman filter as

$$
\hat{\chi}_{L}(t+1 \mid t)=\Phi_{L} \hat{\chi}_{L}(t \mid t-1)+\Lambda_{L}\left[y_{L}(t)-H_{L} \hat{\chi}_{L}(t \mid t-1)\right]
$$

where 


$$
\Lambda_{L}=\Phi_{L} \Pi_{L} H_{L}^{\prime}\left(H_{L} \Pi_{L} H_{L}^{\prime}+R\right)^{-1},
$$

with $\Pi_{L}$ being the non-negative definite solution of the Riccati equation

$$
\Pi_{L}=\Phi_{L} \Pi_{L} \Phi_{L}^{\prime}+\Phi_{L} \Pi_{L} H_{L}^{\prime}\left(H_{L} \Pi_{L} H_{L}^{\prime}+R\right)^{-1} H_{L} \Pi_{L} \Phi_{L}^{\prime}+\Omega_{L} Q \Omega_{L}^{\prime} .
$$

Apparently, the above Riccati equation is dual to that used for the predictor based optimal state feedback regulator discussed by Mita (1985). As a dual of the result given by Mita (1985), we have the following results for the above estimator.

Lemma 4.3: (a) The filter gain matrix $\Lambda_{L}$ can be expressed as

$$
\Lambda_{L}=\left[\begin{array}{c}
\Phi^{L} \Lambda \\
H \Phi^{L-1} \Lambda \\
\vdots \\
H \Phi \Lambda \\
H \Lambda
\end{array}\right]
$$

where $\Lambda$ is the Kalman filter gain matrix (4.16) for the estimation problem without the observation delay, i.e., $L=0$.

(b) For the filter gain matrix (4.39), the error transition matrix $\left(\Phi_{L}-\Lambda_{L} \mathrm{H}_{L}\right)$ is given as

$$
\Phi_{L}-\Lambda_{L} H_{L}=\left[\begin{array}{ccccccc}
\Phi & 0 & \cdot & . & \cdot & 0 & -\Phi^{L} \Lambda \\
I & 0 & \cdot & . & \cdot & 0 & -H \Phi^{L-1} \Lambda \\
0 & I & . & . & . & . & -H \Phi^{L-2} \Lambda \\
\vdots & . & . & . & . & . & \vdots \\
0 & 0 & . & . & \cdot & I & -H \Lambda
\end{array}\right],
$$

The eigenvalues of the matrix $\left(\Phi_{L}-\Lambda_{L} H_{L}\right)$ consist of those of $(\Phi-\Lambda H)$ and $L p$ zeros.

Using the above lemma, we can construct a frequency-shaped Kalman filter which estimates only the minimum-phase state. In the frequency domain, the resulting filter algorithm and its estimation error dynamics can be expressed as follows.

Lemma 4.4: For the estimation problem (4.32), where the matrices $\Phi, \Omega$ and $H$ are defined in (4.4), the optimal one-step ahead prediction of the minimum-phase state $x_{m}(t)$ is generated by the Kalman filter

$$
\hat{x}_{m}(z)=\left[z I-A+z^{-L} K_{L}(z) G_{a}(z) C_{m}\right]^{-1} K_{L}(z) y_{L}(z),
$$

where $K_{L}(z)$ is the frequency-shaped filter gain matrix defined as

$$
K_{L}(z)=A^{L} K(z) \bar{W}_{L}^{-1}(z) .
$$

The matrices $K(z)$ and $\bar{W}_{L}(z)$ in (4.42) are defined in (4.22) and (4.27), respectively. In addition, assume that the disturbance $w(t)$ and the observation noise $v(t)$ are absent. Then it follows from (4.4), (4.32) and (4.41) that the behavior of the estimation error

$$
\tilde{x}_{m}(z)=x_{m}(z)-\hat{x}_{m}(z)
$$

is described by

$$
z \tilde{x}_{m}(z)=A \tilde{x}_{m}(z)-z^{-L} K_{L}(z) G_{a}(z) C_{m} \tilde{x}_{m}(z)
$$

Proof: See Appendix A.

The error dynamics (4.44) can be illustrated as in Fig. 2, where the second term in the right side of (4.44) can be regarded as the estimation error feedback to the open loop error dynamics $z \tilde{x}_{m}(z)=A \tilde{x}_{m}(z)$. We call this feedback system the minimum-phase prediction error feedback (MPF) system. For this feedback system, we can obtain the following result.

Lemma 4.5: Consider the MPF system shown in Fig. 2. The sensitivity matrix at the point corresponding to the output prediction error can be expressed as

$$
\Sigma_{L}^{M P F}(z)=\bar{W}_{L}(z)\left[I+G_{a}(z) C_{m}(z I-A)^{-1} K(z)\right]^{-1},
$$

where $\bar{W}_{L}(z)$ is defined in (4.27).

Proof: The sensitivity matrix at the marked point in Fig. 2 is defined as

$$
\Sigma_{L}^{M P F}(z)=\left[I+z^{-L} G_{a}(z) C_{m}(z I-A)^{-1} K_{L}(z)\right]^{-1} .
$$

Using (4.27) and (4.42), we can rewrite (4.46) as 


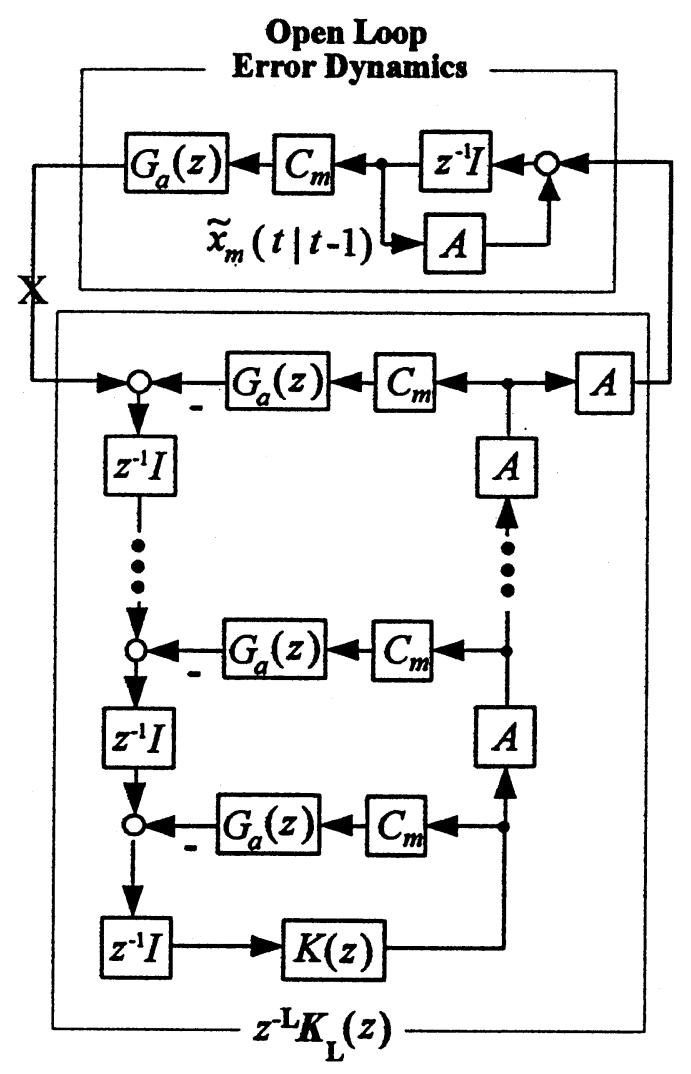

Fig. 2 Structure of the MPF system.

$$
\begin{aligned}
\Sigma_{L}^{M P F}(z) & =\bar{W}_{L}(z)\left[\bar{W}_{L}(z)+z^{-L} G_{a}(z) C_{m}(z I-A)^{-1} K(z)\right]^{-1} \\
& =\bar{W}_{L}(z)\left\{I+G_{a}(z) C_{m}\left[z^{-1} I+\cdots+z^{-L} A^{L-1}+z^{-L} A^{L}(z I-A)^{-1}\right] K(z)\right\}^{-1} .
\end{aligned}
$$

Noting that the matrix identity

$$
z^{-1} I+\cdots+z^{-L} A^{L-1}+z^{-L} A^{L}(z I-A)^{-1}=(z I-A)^{-1}
$$

holds, we can rewrite (4.47) as (4.45).

Comparison of the above result with Proposition 4.1 readily gives the following interpretation of the recovered feedback properties.

Proposition 4.2: The sensitivity matrix recovered by the partial loop recovery technique at the output of the plant coincides with the sensitivity matrix at the output of the MPF system.

\section{RELATION BETWEEN THE TWO TECHNIQUES}

In this section, we clarify the relation between the two LTR techniques discussed in the previous sections. We now need an explicit expression of the all-pass part $G_{a}(z)$. Various methods exist to obtain an expression of $G_{a}(z)$. To discuss the formal application of the LTR procedure, Zhang and Freudenberg (1991) have used a discrete-time version of the decomposition found by Enns (1988) for the continuous-time case. However, their expression is somewhat complicated. Here, we use a simpler expression which clarifies similarities and differences between continuous-time and discrete-time cases. Our expression is summarized as follows.

Lemma 5.1: Assume that the plant (2.1) has $k$ unstable real zeros $q_{1}, q_{2}, \cdots, q_{k}$. Then the plant transfer function matrix $G(z)=C(z I-A)^{-1} B$ can be decomposed as

$$
G(z)=G_{a}(z) G_{m}(z)
$$

where $G_{a}(z)$ and $G_{m}(z)$ are all-pass part and minimum phase part, respectively. The minimum phase part is given by

$$
G_{m}(z)=C_{m}(z I-A)^{-1} B,
$$

where the matrix $C_{m}$ is defined by

$$
C_{m}=J_{k} O_{k},
$$


where

$$
J_{i}=I-\left(q_{i}+1\right) \eta_{i} \eta_{i}^{\prime}, \quad O_{i}=J_{i-1} O_{i-1}-\left(q_{i}-\frac{1}{q_{i}}\right) \eta_{i} \xi_{i}^{\prime}, \quad i=1,2, \cdots, k,
$$

with the initial conditions $O_{0}=C, J_{0}=I$ and $\xi_{i}$ and $\eta_{i}$ are vectors satisfying the relation

$$
\left[\begin{array}{ll}
\xi_{i}^{\prime} & \eta_{i}^{\prime}
\end{array}\right]\left[\begin{array}{cc}
q_{i} I-A & -B \\
-C & 0
\end{array}\right]=0, \quad \eta_{i}^{\prime} \eta_{i}=1
$$

The all-pass part $G_{a}(z)$ is given by

$$
G_{a}(z)=G_{a k}(z) G_{a(k-1)}(z) \cdots G_{a 1}(z),
$$

where

$$
G_{a i}(z)=\left(I-\eta_{i} \eta_{i}^{\prime}\right)+\frac{z-q_{i}}{1-q_{i} z} \eta_{i} \eta_{i}^{\prime}
$$

and satisfies

$$
G_{a}^{\prime}\left(z^{-1}\right) G_{a}(z)=I
$$

Using the above result, we construct a realization of $G_{a}(z)$. First, let $\left\{A_{a i}, B_{a i}, C_{a i}, D_{a i}\right\}$ denote a realization of $G_{a i}(z)$ defined by (5.7). We choose

$$
A_{a i}=\frac{1}{q_{i}}, \quad B_{a i}=\eta_{i}^{\prime}, \quad C_{a i}=\frac{1}{q_{i}}\left(q_{i}-\frac{1}{q_{i}}\right) \eta_{i}, \quad D_{a i}=I-\left(1+\frac{1}{q_{i}}\right) \eta_{i} \eta_{i}^{\prime} .
$$

In addition, let $\left\{\bar{A}_{i}, \bar{B}_{i}, \bar{C}_{i}, \bar{D}_{i}\right\}$ denote a realization of $G_{a 1}(z) G_{a 2}(z) \cdots G_{a i}(z)$. Noting that $G_{a 1}(z) G_{a 2}(z)$ $\cdots G_{a(i+1)}(z)$ can be regarded as a series connection of $G_{a 1}(z) G_{a 2}(z) \cdots G_{a i}(z)$ and $G_{a(i+1)}(z)$, we can easily show that the following recursive relation

$$
\begin{aligned}
& \bar{A}_{i+1}=\left[\begin{array}{cc}
A_{a(i+1)} & O \\
\bar{B}_{i} C_{a(i+1)} & \bar{A}_{i}
\end{array}\right], \quad \bar{B}_{i+1}=\left[\begin{array}{c}
B_{a(i+1)} \\
\bar{B}_{i} D_{a(i+1)}
\end{array}\right], \\
& \bar{C}_{i+1}=\left[\begin{array}{ll}
\bar{D}_{i} C_{a(i+1)} & \bar{C}_{i}
\end{array}\right], \quad \bar{D}_{i+1}=\bar{D}_{i} D_{a(i+1)} .
\end{aligned}
$$

holds for $i=1,2, \cdots, k-1$ with the initial conditions

$$
\overline{A_{1}}=A_{a 1}, \quad \bar{B}_{1}=B_{a 1}, \quad \bar{C}_{1}=C_{a 1}, \quad \bar{D}_{1}=D_{a 1} .
$$

Apparently, a realization $\left\{A_{a}, B_{a}, C_{a}, D_{a}\right\}$ of $G_{a}(z)$ is given by $\left\{\bar{A}_{k}, \bar{B}_{k}, \bar{C}_{k}, \bar{D}_{k}\right\}$.

For the above realizations, we first give the following result.

Lemma 5.2: Assume that the plant (2.1) has $\mathrm{k}$ unstable real zeros $q_{1}, q_{2}, \cdots, q_{k}$. Take the realization $\left\{\bar{A}_{k}, \bar{B}_{k}, \bar{C}_{k}, \bar{D}_{k}\right\}$ obtained from (5.1) as a realization of $G_{a}(z)$. Define the matrix

$$
\Theta \triangleq\left[\begin{array}{llllll}
I & q_{k} \xi_{k} & q_{k-1} \xi_{k-1} & \cdots & q_{1} \xi_{1}
\end{array}\right]^{\prime}
$$

Then the following matrix identities hold:

$$
\Phi \Theta=\Theta A, \quad \Theta B=\Gamma, \quad H \Theta=C
$$

Proof: The proof is based on induction on the indexes assigned to the unstable zeros. An outline is given in Appendix B.

Using the above lemma, we find the following simple result for the relation between the Riccati equations (2.8) and (4.17).

Lemma 5.3: Suppose that the assumptions made in Lemma 5.2 hold. In addition, assume that the covariance matrix of the disturbance $w(t)$ is given by $Q=B B^{\prime}$. Then, the matrix defined by

$$
\Pi_{P} \triangleq \Theta P \Theta^{\prime}
$$

where the matrix $P$ is a positive definite solution of the Riccati equation (2.8), is a non-negative definite solution of the Riccati equation (4.17), which is related to the stochastic model (4.2) based on the decomposed transfer function matrix $G_{m}(z) G_{a}(z)$.

Proof: First, notice that the matrix $\Pi_{P}$ is non-negative definite since the matrix $P$ is positive definite. Substituting (5.14) into the right side of (4.17) and using (5.14), we have

$$
\Phi \Pi_{P} \Phi^{\prime}-\Phi \Pi_{P} H^{\prime}\left(H \Pi_{P} H^{\prime}+R\right)^{-1} H \Pi_{P} \Phi^{\prime}+\Gamma \Gamma^{\prime}=\Phi\left[A P A^{\prime}-A P C^{\prime}\left(C P C^{\prime}+R\right)^{-1} C P A^{\prime}+B B^{\prime}\right] \Phi^{\prime},
$$


Noting that the matrix $P$ satisfy the Riccati equation (2.8), the above matrix is reduced to $\Theta P \Theta^{\prime}=\Pi_{P}$, which implies that $\Pi_{P}$ satisfies the Riccati equation (4.17).

For the filter gain matrices related to (4.17), we have the following result sing the above lemmas.

Lemma 5.4: Suppose that the assumptions made in Lemma 5.3 bold. Consider the prediction type Kalman filter (4.15) for the stochastic model (4.2) based on the all-pass/minimum phase decomposition. Then the optimal filter gain matrices $K_{m}$ and $K_{a}$, defined in (4.18), can be expressed as

$$
K_{m}=K, \quad K_{a}=\Xi K,
$$

where

$$
\Xi \triangleq\left[\begin{array}{llll}
q_{m} \xi_{m} & q_{m-1} \xi_{m-1} & \cdots & q_{1} \xi_{1}
\end{array}\right]^{\prime}
$$

and $K$ is the filter gain matrix (2.7) defined by the solution $P$ of the Riccati equation (2.8).

Proof: Using (5.13) in (4.16), we have

$$
\begin{aligned}
{\left[\begin{array}{ll}
K_{m}^{\prime} & K_{a}^{\prime}
\end{array}\right]^{\prime} } & =\Phi \Pi_{P} H^{\prime}\left(R+H \Pi_{P} H^{\prime}\right)^{-1}=\Phi \Theta P \Theta^{\prime} H^{\prime}\left(R+H \Theta P \Theta^{\prime} H^{\prime}\right)^{-1} \\
& =\Theta A P C^{\prime}\left(R+C P C^{\prime}\right)^{-1}=\Theta K .
\end{aligned}
$$

Comparing the matrix (5.12) with (5.17), we can rewrite (5.18) as

$$
\left[\begin{array}{ll}
K_{m}^{\prime} & K_{a}^{\prime}
\end{array}\right]=\left[\begin{array}{ll}
I & \Xi^{\prime}
\end{array}\right]^{\prime} K,
$$

which proves (5.16).

Lemma 5.5: As a realization $\left\{A_{a}, B_{a}, C_{a}, D_{a}\right\}$ for the all-pass part $G_{a}(z)$, take $\left\{\bar{A}_{k}, \bar{B}_{k}, \bar{C}_{k}, \bar{D}_{k}\right\}$ defined by the recursive relation (5.10). Then the following matrix identity holds for the filter gain matrices satisfying (5.16).

$$
C_{a}\left(z I-A_{a}\right)^{-1} K_{a}+G_{a}(z) C_{m}(z I-A)^{-1} K_{m}=C(z I-A)^{-1} K
$$

Proof: See Appendix C.

Using the above lemma together with Propositions 3.1 and 4.1, we can obtain the following result, which clarifies the relation between the two LTR techniques.

Proposition 5.1: Assume that the covariance matrix of the disturbance $w(t)$ is given by $Q=B B^{\prime}$. Then

$$
\Sigma_{L}^{*}(z)=S_{L}^{*}(z)
$$

where $S_{L}^{*}(z)$ and $\Sigma_{L}^{*}(z)$ are defined in (3.6) and (4.29), respectively.

Proof: Take $\left\{\bar{A}_{k}, \bar{B}_{k}, \bar{C}_{k}, \bar{D}_{k}\right\}$ defined by the recursive relation (5.10) as a realization of the all-pass part $G_{a}(z)$. Then, we can use the preceding lemmas. Notice that the frequency-shaped filter gain matrix $K(z)$ in the sensitivity matrix (4.29) is defined in (4.22). So, for the filter gain matrices satisfying (5.16), we can rewrite (4.29) as

$$
\Sigma_{L}^{*}(z)=\left[\bar{W}_{L}(z)+C_{a}\left(z I-A_{a}\right)^{-1} \Xi K\right]\left[I+C_{a}\left(z I-A_{a}\right)^{-1} \Xi K+G_{a}(z) C_{m}(z I-A)^{-1} K\right]^{-1} .
$$

where $\tilde{W}_{L}(z)$ is defined in (3.7). It follows from Lemma 5.5 that

$$
\begin{aligned}
& C_{a}\left(z I-A_{a}\right)^{-1} \Xi K=\left[C-G_{a}(z) C_{m}\right](z-A)^{-1} K, \\
& C_{a}\left(z I-A_{a}\right)^{-1} \Xi K+G_{a}(z) C_{m}(z I-A)^{-1} K=C(z I-A)^{-1} K .
\end{aligned}
$$

Substituting (5.23) into (5.22), we readily obtain (5.21).

The above result shows that, for the common choice of the disturbance and observation noise matrices, the feedback properties achieved by the two LTR techniques coincide. Since the partial LTR procedure is plausible for dealing with non-minimum phase plants, the above result provides a clear system-theoretic justification for enforcement of the conventional LTR procedure. It should be noted that this fact does not make the partial LTR technique useless for practical applications. The partial LTR technique provides more freedom in selecting a target than the conventional LTR technique. In the following, we present a simple technique exploiting this freedom.

\section{A TARGET WITH MORE FREEDOM}

Replace (4.2) by the stochastic model

$$
\chi(t+1)=\Phi \chi(t)+\Gamma u(t)+\omega(t), \quad y(t)=H \chi(t)+v(t),
$$

where

$$
\omega(t)=\left[\omega_{m}^{\prime}(t) \quad \omega_{a}^{\prime}(t)\right]^{\prime}
$$

is the zero mean white noise process with the covariance matrix

$$
\Omega_{p}=\operatorname{diag}\left[\begin{array}{ll}
\Omega_{m} & \Omega_{a}
\end{array}\right] \text {. }
$$


Since the model (4.2) is obtained by taking $\Omega_{m}=Q$ and $\Omega_{a}=0$ in (5.26), the above model is more general than (4.2). For the model (5.24), the target sensitivity matrix that can be recovered by the partial LTR procedure is obtained by replacing the filter gain matrix in (4.22) by that for (5.24). Note that the above stochastic model can be rewritten as

$$
\begin{aligned}
& x_{m}(t+1)=A x_{m}(t)+B u(t)+\omega_{m}(t), \\
& y(t)=G_{a}(z) C_{m} x_{m}(t)+C_{a}\left(z I-A_{a}\right)^{-1} \omega_{a}(t)+v(t) .
\end{aligned}
$$

Furthermore, it can easily be checked that the observation equation (5.28) is statistically equivalent to

$$
y(t)=G_{a}(z) C_{m} x_{m}(t)+R_{a}(z) \varepsilon(t),
$$

where $\varepsilon(t)$ is a zero mean white noise process with the unit covariance matrix, and the shaping filter $R_{a}(z)$ satisfies the spectral factorization

$$
R_{a}(z) R_{a}^{\prime}\left(z^{-1}\right)=R+C_{a}\left(z I-A_{a}\right)^{-1} \Omega_{a}\left(z^{-1} I-A_{a}^{\prime}\right)^{-1} C_{a}^{\prime} .
$$

Using the standard state space result (e.g., Anderson and Moore, 1990), we can express $R_{a}(z)$ as

$$
R_{a}(z)=\left[I+C_{a}\left(z I-A_{a}\right)^{-1} A_{a} P_{a} C_{a}^{\prime}\left(C_{a} P_{a} C_{a}^{\prime}+R\right)^{-1}\right]\left(C_{a} P_{a} C_{a}^{\prime}+R\right)^{1 / 2},
$$

where $P_{a}$ is the non-negative solution of the Riccati equation

$$
P_{a}=A_{a} P_{a} A_{a}^{\prime}+A_{a} P_{a} C_{a}^{\prime}\left(C_{a} P_{a} C_{a}^{\prime}+R\right)^{-1} C_{a} P_{a} A_{a}^{\prime}+\Omega_{a} .
$$

Because the matrix $A_{a}$ is stable and the pair $\left(C_{a}, A_{a}\right)$ is observable, the non-negative definite solution of (5.32) exists so that the existence of the spectral factor $R_{a}(z)$ is gnaranteed. Note that $R_{a}(z)$ defined by (5.31) is minimum phase.

For (5.27) and (5.29), we can consider an estimation problem estimating only the minimum phase state $x_{m}(t)$ under colored observation noise. The dynamics of the shaping filter $R_{a}(z)$ depends on the covariance matrix $\Omega_{a}$. The principal gains of $R_{a}(z)$ provide useful information to choose $\Omega_{a}$ so as to achieve the desired feedback property. Note that the principal gains can be obtained directly from the right side of (5.30) without solving the Riccati equation (5.32).

\section{NUMERICAL EXAMPLE}

Consider the single-input single-output minimum phase continuous-time plant described by the transfer function

$$
G(s)=\frac{s+10}{(s+1)(s+0.5 \pm 1.2 j)} .
$$

Discretizing with a zeroth order holder and sampling periord $\mathrm{T}=0.1(\mathrm{sec}$.), we have a non-minimum phase discrete-time model of (6.1), with the single unstable zero $z=-1.2813$. A state space representation for the decomposed transfer function corresponding to (3.4) and (4.1) is given by

$$
\begin{aligned}
& A=\left[\begin{array}{ccc}
2.7636 & -2.6139 & 0.8187 \\
1 & 0 & 0 \\
0 & 1 & 0
\end{array}\right], \quad B=\left[\begin{array}{l}
1 \\
0 \\
0
\end{array}\right], \quad C_{m}=\left[\begin{array}{llll}
0.0080 & 0.0033 & -0.0023
\end{array}\right], \\
& A_{a}=-0.7805, \quad B_{a}=0.3903, \quad C_{a}=1, \quad D_{a}=0.28188 .
\end{aligned}
$$

Note that the all-pass part is scalar since (6.1) contains the single unstable zero.

To illustrate the design freedom provided by the partial LTR technique, we construct a target by using the model (5.24) with a special choice of the variances

$$
\Omega_{a}=\mu R, \quad \Omega_{m}=B B^{\prime},
$$

where $\mu$ is a new positive scalar parameter for tuning the target feedback property. Defining the spectral factorization

$$
\psi_{a}(z) \psi_{a}\left(z^{-1}\right)=1+\frac{\mu}{\left(z-A_{a}\right)\left(z^{-1}-A_{a}\right)},
$$

we can write the spectral factor in (5.30) for the present case as

$$
R_{a}(z)=R^{1 / 2} \psi_{a}(z)
$$

Introducing a new positive scalar parameter $\sigma$, we take the variance $R$ as 


$$
R=\frac{\sigma}{\psi^{2}(1)} \text {. }
$$

If the two new parameters $\mu$ and $\sigma$ are specified, we can determine the filter gain matrix by solving the Riccati equation for the corresponding variances $R$ and $\Omega_{a}$. Note that $R_{a}(1)=\sigma$ holds and that the parameter $\mu$ determines the shape of the frequency response of $R_{a}(z)$. Keeping dc gain constant by fixing $\sigma$, we can manipulate the shape of $R_{a}(z)$ by the parameter $\mu$. The gain characteristic of the shaping filter $R_{a}(z)$ is shown in Fig. 3. As the parameter $\mu$ increases, the gain in the high frequency region increases with no significant influence in the low frequency region. For $L=1$ and $\sigma=0.001$, the gain characteristics of the sensitivity function and the complementary sensitivity function of the target for several values of the parameter $\mu$ are shown in Fig. 4 and 5, respectively. The corresponding results for $L=2$ are shown in Figs. 6 and 7. As is expected from the gain characteristic of $R_{a}(z)$ shown in Fig. 3, the influence of the parameter $\mu$ is significant near the Nyquist frequency. This feature is particularly useful to enhance the robustness against high frequency uncertainties, as seen from the plots for the complementary sensitivity functions.

\section{CONCLUSIONS}

For discrete-time non-minimum phase plants with feedback delays, we have discussed the two LTR techniques based on the Riccati equation formalism. The results give a clear system-theoretic interpretation to the

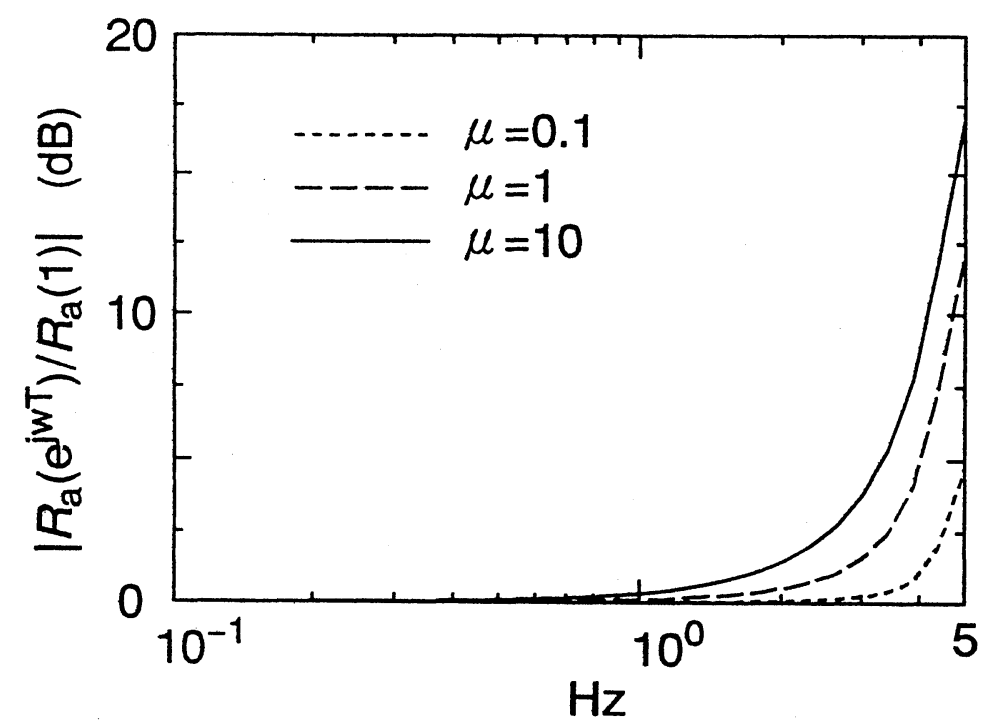

Fig. 3 Gain characteristic of $R_{\mathrm{a}}(z)$.

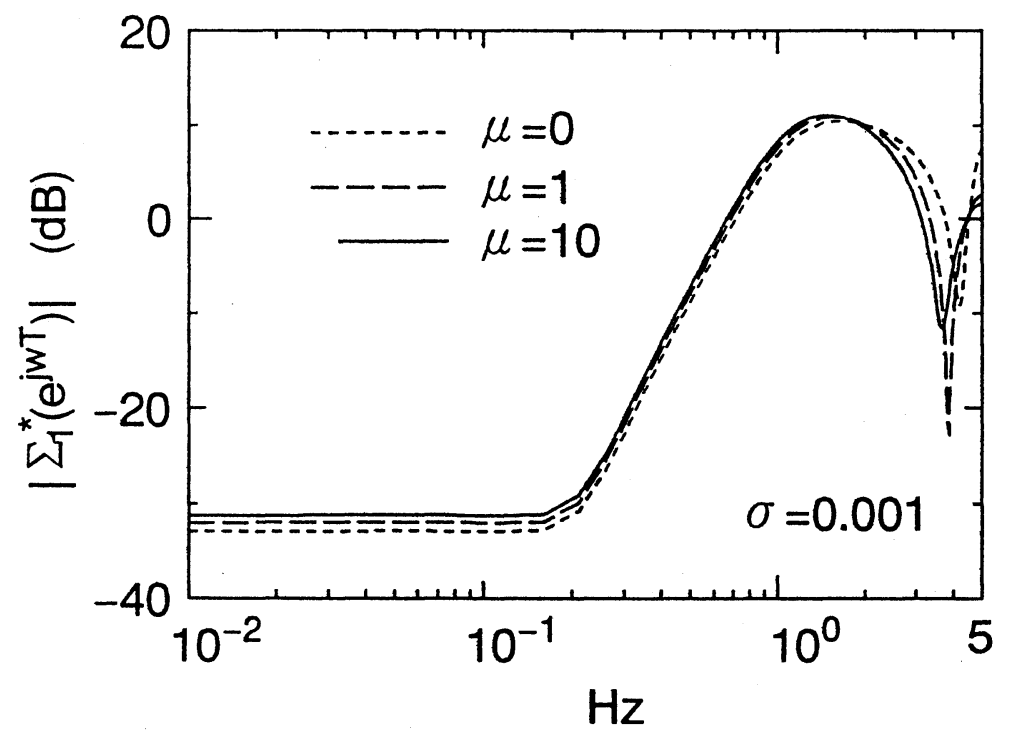

Fig. 4 Sensitivity characteristic of the target for $L=1$. 


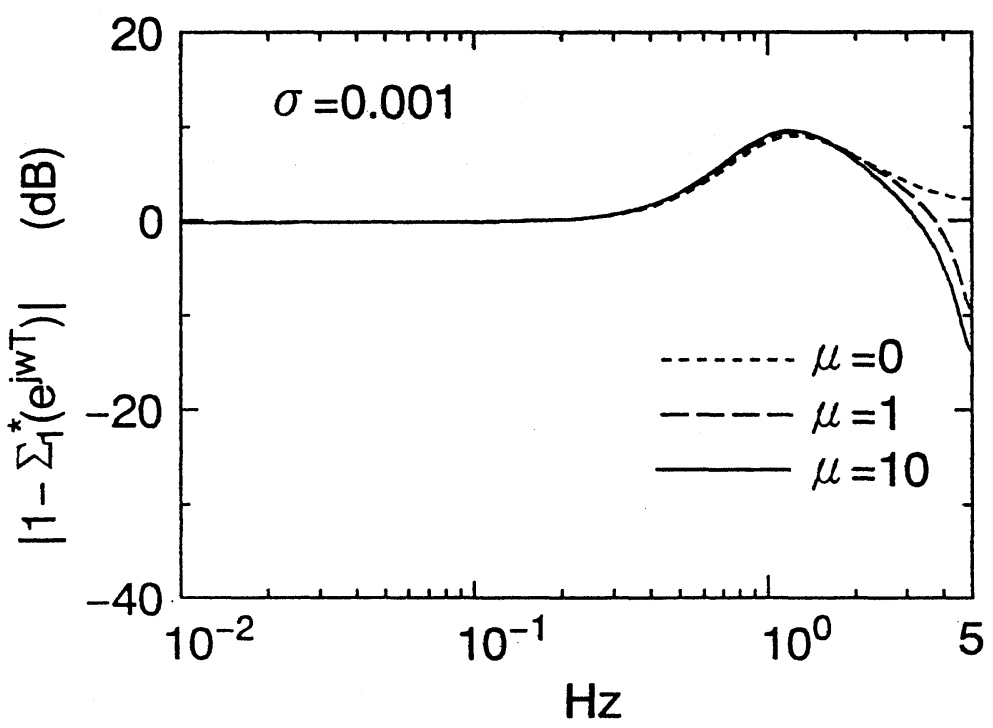

Fig. 5 Complementary sensitivity characteristic of the target for $L=1$.

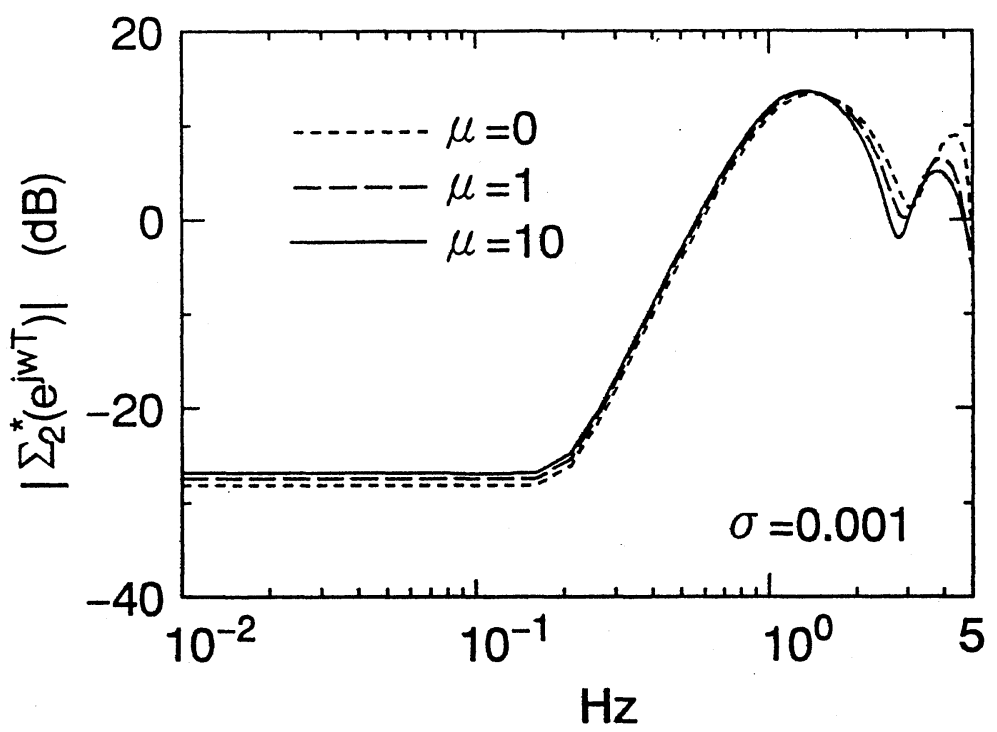

Fig. 6 Sensitivity characteristic of the target for $L=2$.

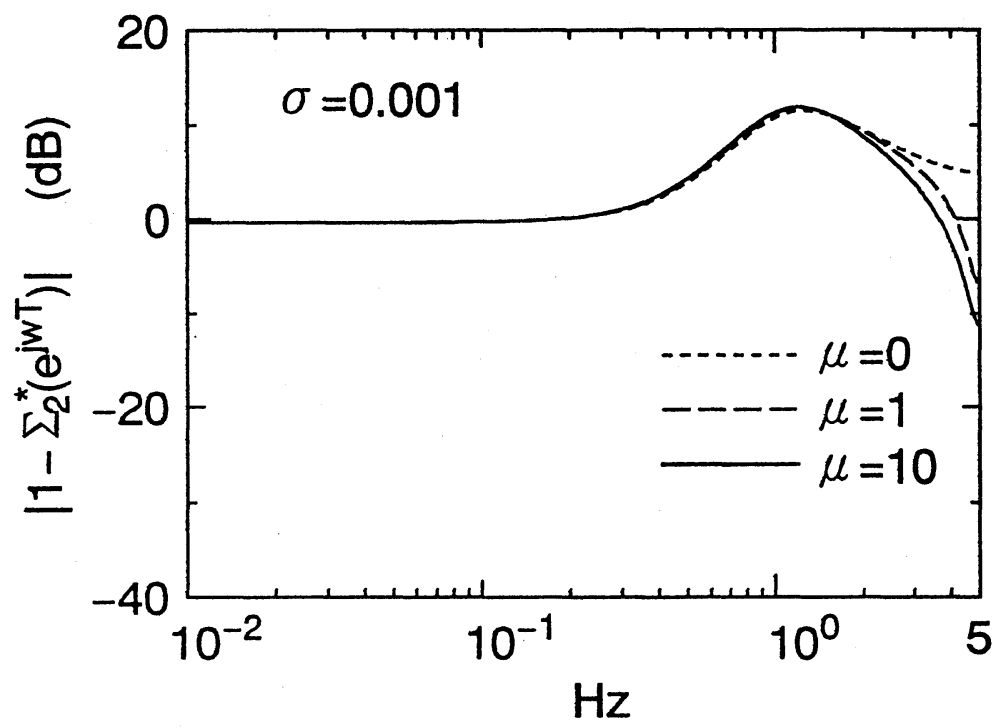

Fig. 7 Complementary sensitivity characteristic of the target for $L=2$. 
formal application of the conventional LTR procedure and justifies its use for a class of non-minimum phase plants. We have also pointed out that the partial LTR technique provides more design freedom in shaping a target property via the selection of a disturbahce model. As an example to exploit the design freedom, we have proposed a simple technique for the partial LTR. A numerical example has shown that the proposed technique is useful to enhance the robustness against uncertainties near the Nyquist frequency without significant change in low frequency characteristics.

\section{REFERENCES}

Anderson, B. D. O., and Moore, J. B., 1990, Optimal Control: Linear Quadratic Methods, Prentice-Hall.

Astrom, K. J., Hagander, P., and Sternby, J., 1984, Zeros of sampled systems, Automatica, 20, 31-38.

Doyle, J. C., and Stein, G., 1979, Robustness with observers, IEEE Trans. Automat. Control, 24, 607-611.

Enns, D., 1984, Model reduction for control system design. Ph.D. Thesis, Stanford Univ.

Halevi, Y., and Ray, A., 1988, Integrated communication and control systems: Part I-Analysis, Journal of Dynamic Systems, Measurement, and Control, 110, 367-373.

Ishihara, T., and Takeda, H., 1986, Loop transfer recovery techniques for discrete-time optimal regulators using prediction estimators, IEEE Trans. Automat. Control, 31, 1149-1151.

Ishihara, T., 1988, Sensitivity properties of a class of discrete-time LQG controllers with computation delays, Systems and Control Letters, 11, 299-307.

Ishihara, T., Watanabe, T., Zheng, L. A., and Inooka, H., 1992, A discrete-time doubly coprime factorization based on the predictor-based LQG controller, Int. J. Contr., 55, 1201-1219, 1992.

Ishihara, T., 1994, Loop transfer recovery techniques for discrete-time non-minimum phase plants. Trans. Society of Instrument and Control Engineers, 30, 1172-1177. (in Japanese)

Kimura, H., 1982, Perfect and subperfect regulation in linear multivariable control systems, Automatica, 18, 125-145.

Kwakernaak, 1969, Optimal low sensitivity linear feedback systems, Automatica, 5, 279--286.

Maciejowski, J., 1985, Asymptotic recovery for discrete-time systems. IEEE Trans. Automat. Control, 30, 602-605.

Mita, T., 1985, Optimal digital feedback control systems counting computation time of control laws. IEEE Trans. Automat. Control, 30, 542-548.

Moore, J. B. and Xia, L., 1987, Loop recovery and robust state feedback designs. IEEE Trans, Automat. Control, 32, 512517.

Saberi, A., Chen, B. M., and Sannuti, P., 1991, Theory of LTR for nonminimum phase systems, recoverable target loops, recovery in a subspace, Part 1 . Analysis, Int. J. Contr., 53, 1067-1115.

Shaked, U., 1985, Explicit solution to the singular discrete-time stationary filtering problem, IEEE Trans. Automat. Contr., $30,34-47$.

Zhang, Z., and Freudenberg, J. S., 1990, Loop transfer recovery for nonminimum phase plants. IEEE Trans. Automat. Control, 35, 547-553.

Zhang, Z., and Freudenberg, J. S., 1991, On discrete-time loop transfer recovery, Proceedings of the 1991 American Control Conference, 2214-2219.

\section{APPENDICES}

\section{A. Proof of Lemma 4.4}

The elements of the optimal estimates generated by (4.36) are given by

$$
\begin{aligned}
& \hat{\chi}(t+1 \mid t)=\Phi \hat{\chi}(t \mid t-1)+\Phi^{L} \Lambda\left[y_{L}(t)-\hat{y}_{L}(t \mid t-1],\right. \\
& \hat{y}_{1}(t+1 \mid t)=H \hat{\chi}(t \mid t-1)+H \Phi^{L-1} \Lambda\left[y_{L}(t)-\hat{y}_{L}(t \mid t-1)\right], \\
& \hat{y}_{j}(t+1 \mid t)=\hat{y}_{j-1}(t \mid t-1)+H \Phi^{L-j} \Lambda\left(y_{L}(t)-\hat{y}_{L}(t \mid t-1)\right], \quad j=2,3, \cdots, L .
\end{aligned}
$$

where $\Lambda$ is the optimal filter gain matrix for $L=0$. Taking the $\mathrm{z}$ transforms of $(\mathrm{A} \cdot 1)$ and eliminating $\hat{y}_{j}(z)$ $(j=1,2, \cdots, L-1)$, we obtain

$$
z^{L} \hat{y}_{L}(z)=H \hat{x}(z)+\left(H \Phi^{L-1} \Lambda+z H \Phi^{L-2} \Lambda+\cdots+z^{L-1} H \Lambda\right)\left[y(z)-\hat{y}_{L}(z)\right] .
$$

Note that the matrix $\Phi^{j}$ can be written as

$$
\Phi^{j}=\left[\begin{array}{cc}
A^{j} & 0 \\
\Delta_{j} & A_{a}^{j}
\end{array}\right],
$$

where

$$
\Delta_{j+1}=A_{a} \Delta_{j}+B_{a} C_{m} A^{j} .
$$

Using $(\mathrm{A} \cdot 3)$ in the first equation in $(\mathrm{A} \cdot 1)$, we obtain

$$
z \hat{x}_{m}(z)=A \hat{x}_{m}(z)+A^{L} K_{m}\left[y_{L}(z)-\hat{y}_{L}(z)\right],
$$




$$
z \hat{x}_{a}(z)=A_{a} \hat{x}_{a}(z)+B_{a} C_{m} \hat{x}_{m}(z)+\left(\Delta_{L} K_{m}+A_{a}^{L} K_{a}\right)\left[y_{L}(z)-\hat{y}_{L}(z)\right]
$$

Noting that

$$
\begin{aligned}
& H \hat{\chi}(z)=D_{a} C_{m} \hat{x}_{m}(z)+C_{a} \hat{x}_{a}(z), \\
& H \Phi^{j} \Lambda=\left(D_{a} C_{m} A^{j}+C_{a} \Delta_{j}\right) K_{m}+C_{a} A_{a}^{j} K_{a},
\end{aligned}
$$

we can rewrite (A.2) as

$$
z^{L} \hat{y}_{L}(z)=G_{a}(z) C_{m} \hat{x}_{m}(z)+T_{L}(z)\left[y(z)-\hat{y}_{L}(z)\right]
$$

where

$$
\left.\left.T_{L}(z)=C_{a}\left(z I-A_{a}\right)^{-1}\left(\Delta_{L} K_{m}+A_{a}^{L} K_{a}\right)+\sum_{j=0}^{L-1}\left[D_{a} C_{m} A^{j}+C_{a} \Delta_{j}\right) K_{m}+C_{a} \Delta_{j}\right) K_{m}+C_{a} A_{a}^{j} K_{a}\right] z^{L-j-1} .
$$

After some simple matrix calculations, we obtain

$$
T_{L}(z)=z^{L} C_{a}\left(z I-A_{a}\right)^{-1} K_{a}+G_{a}(z) C_{m} A^{L-1} K_{m}+z G_{a}(z) C_{m} A^{L-2} K_{m}+\cdots+z^{l-1} G_{a}(z) C_{m} K_{m} .
$$

Eliminating $\hat{y}_{L}(z)$ from (A.5) by using (A.9), we have

$$
z \hat{x}_{m}(z)=\left\{A-A^{L} K_{m}\left[z^{L} I+T_{L}(z)\right]^{-1} G_{a}(z) C_{m}\right\} \hat{x}_{m}(z)+A^{L} K_{m}\left[z^{L}+T_{L}(z)\right]^{-1} z^{L} y_{L}(z) .
$$

It follows from (A.11) that

$$
z^{L} I+T_{L}(z)=z^{L} W_{L}(z)\left[I+C_{a}\left(z I-A_{a}\right)^{-1} K_{a}\right] .
$$

Substituting (A.14) into (A.13), we obtain

$$
z \hat{x}_{m}(z)=\left\{A-z^{-L} A^{L} K(z) W_{L}^{-L}(z) G_{a}(z) C_{m}\right\} \hat{x}_{m}(z)+A^{L} K(z) W_{L}^{-1}(z) y_{L}(z),
$$

which is equivalent to the Kalman filter algorithm (4.39) with the frequency-shaped filter gain matrix (4.40). The error dynamics (4.42) readily follow from (A.15) by noting that $z \tilde{x}_{m}(z)=A \tilde{x}_{m}(z)$ and $y_{L}(z)=z^{-L} G_{a}(z) C_{m} x_{m}(z)$.

\section{B. Proof of Lemma 5.2}

Note that the second relation $\Theta B=\Gamma$ readily follows since the relation (5.5) implies that $\xi_{i}^{\prime} B=0$. To prove the remaining two relations, we consider the decomposition

$$
G(z)=G_{a 1}(z) G_{a 2}(z) \cdots G_{a i}(z) J_{i} O_{i}(z I-A)^{-1} B,
$$

and define a realization $\left\{\Phi_{i}, \Gamma_{i}, H_{i}\right\}$ of $G(z)$ as

$$
\Phi_{i}=\left[\begin{array}{cc}
A & O \\
\bar{B}_{i} C_{i} & \bar{A}_{i}
\end{array}\right], \quad \Gamma_{i}=\left[\begin{array}{c}
B \\
0
\end{array}\right], \quad H_{i}=\left[\begin{array}{ll}
\bar{D}_{i} C_{i} & \bar{C}_{i}
\end{array}\right] .
$$

Although details are omitted, we can show by induction on the index $i$ that

$$
\Phi_{i} \Theta_{i}=\Theta_{i} A, \quad H_{i} \Theta_{i}=C,
$$

where

$$
\Theta_{i} \triangleq\left[\begin{array}{lllll}
I & q_{i} \xi_{i} & q_{i-1} \xi_{i-1} & \cdots & q_{1} \xi_{1}
\end{array}\right]^{\prime}
$$

Noting that $\Phi_{k}=\Phi, H_{k}=H$ and $\Theta_{k}=\Theta$ hold by definition, the relation (B.3) implies that $\Phi \Theta=\Theta A$ and $H \Theta=C$.

\section{Proof of Lemma 5.5}

By induction, we prove

$$
\bar{C}_{i}\left(z I-\bar{A}_{i}\right)^{-1} \Xi_{i} K+G_{a 1}(z) G_{a 2}(z) \cdots G_{a i}(z) J_{i} O_{i}(z I-A)^{-1} K=C(z I-A)^{-1} K,
$$

where the matrices $\bar{A}_{i}$ and $\bar{C}_{i}$ are defined recursively by (5.10) and

$$
\Xi_{i} \triangleq\left[\begin{array}{llll}
q_{i} \xi_{i} & q_{i-1} \xi_{i-1} & \cdots & q_{1} \xi_{1}
\end{array}\right]^{\prime} .
$$

By simple calculations, we can show that

$$
\begin{aligned}
& q_{i} C_{a i}\left(z I-A_{a i}\right)^{-1}=\frac{q_{i}^{2}-1}{q_{i} z-1} \eta_{i}, \\
& G_{a i}(z) J_{i} O_{i}=J_{i-1} O_{i-1}-\frac{q_{i}^{2}-1}{q_{i} z-1} \eta_{i} \xi_{i}^{\prime}(z I-A) .
\end{aligned}
$$

Note that $C_{0}=C$ and $\Xi_{1}=q_{1} \xi_{1}$. Using (C.3) and (C.4) for $i=1$, we can immediately check that the relation 
(C.1) holds for $i=1$. Assume that (C.1) holds for $i$. Simple calculation yields

$$
\begin{aligned}
\bar{C}_{i+1}\left(z I-\bar{A}_{i+1}\right)^{-1} \Xi_{i+1} K= & {\left[\begin{array}{ll}
\bar{D}_{i} C_{a(i+1)} & J_{i} O_{i}
\end{array}\right]\left[\begin{array}{cc}
z I-A_{a(i+1)} & 0 \\
-\bar{B}_{i} C_{a(i+1)} & z I-\bar{A}_{i}
\end{array}\right]^{-1}\left[\begin{array}{c}
q_{i+1} \xi_{i+1} \\
\Xi_{i}
\end{array}\right] K } \\
= & {\left[\bar{C}_{i}\left(z I-\bar{A}_{i}\right)^{-1} \bar{B}_{i}+\bar{D}_{i}\right] C_{a(i+1)}\left(z-A_{a(i+1)}\right)^{-1} q_{i+1} \xi_{i+1}^{\prime} K } \\
& +\bar{C}_{i}\left(z I-\bar{A}_{i}\right)^{-1} \Xi_{i} K .
\end{aligned}
$$

Noting that $\left\{\bar{A}_{i}, \bar{B}_{i}, \bar{C}_{i}, \bar{D}_{i}\right\}$ is a realization of $G_{a 1}(z) G_{a 2}(z) \cdots G_{a i}(z)$ and using (C.4) and (C.5), we have

$$
\begin{aligned}
& \bar{C}_{i+1}\left(z I-\bar{A}_{i+1}\right)^{-1} \Xi_{i+1} K+G_{a 1}(z) G_{a 2}(z) \cdots G_{a(i+1)}(z) J_{i+1} O_{i+1}(z I-A)^{-1} K \\
&= \bar{C}_{i}\left(z I-\bar{A}_{i}\right)^{-1} \Xi_{i} K+G_{a 1}(z) \cdots G_{a i}(z) J_{i} O_{i}(z I-A)^{-1} K \\
& \quad+G_{a 1}(z) \cdots G_{a i}(z)\left[C_{a(i+1)}\left(z-A_{a(i+1)}\right)^{-1} q_{i+1}-\frac{q_{i+1}^{2}-1}{q_{i+1} z-1} \eta_{i+1}\right] \xi_{i+1}^{\prime} K .
\end{aligned}
$$

Using (C.3) and the assumption that (C.1) holds for $i$, we can readily see that (C.7) reduces to $C(z I-A)^{-1} K$. Consequently, (C.1) holds for $i=1,2, \cdots, k$. The expression (C.1) with $i=k$ is equivalent to (5.20) with $K_{a}$ and $K_{m}$ given by (5.16) in Lemma 5.4. 